\title{
Médiévales
}

Langues, Textes, Histoire

46 | printemps 2004

Éthique et pratiques médicales

\section{Éthique des consilia et de la consultation : à propos de la cohésion morale de la profession médicale (XIII ${ }^{\mathrm{e}}$-XIV ${ }^{\mathrm{e}}$ siècles)}

Traduit de l'italien par Marilyn Nicoud

\section{Chiara Crisciani}

\section{(2) OpenEdition}

\section{Journals}

Édition électronique

URL : https://journals.openedition.org/medievales/989

DOI : 10.4000/medievales.989

ISSN : 1777-5892

\section{Éditeur}

Presses universitaires de Vincennes

Édition imprimée

Date de publication : 1 juin 2004

Pagination : 23-44

ISBN : 2-84292-151-8

ISSN : 0751-2708

\section{Référence électronique}

Chiara Crisciani, «Éthique des consilia et de la consultation : à propos de la cohésion morale de la profession médicale (xIII-xIV ${ }^{e}$ siècles) », Médiévales [En ligne], 46 I printemps 2004, mis en ligne le 02 mars 2006, consulté le 23 avril 2022. URL : http://journals.openedition.org/medievales/989 ; DOI : https://doi.org/10.4000/medievales.989

Ce document a été généré automatiquement le 23 avril 2022.

Tous droits réservés 


\title{
Éthique des consilia et de la consultation : à propos de la cohésion morale de la profession médicale (XIII ${ }^{\mathrm{e}}-\mathrm{XIV}^{\mathrm{e}}$ siècles) ${ }^{*}$
}

Traduit de l'italien par Marilyn Nicoud

\author{
Chiara Crisciani
}

Depuis le $\mathrm{xIII}^{\mathrm{e}}$ siècle ${ }^{1}$, la compétence doctrinale, acquise et légitimée auprès des institutions universitaires, est à la base de l'autorité nouvelle et du prestige plus solide qui permettent au médecin de se mouvoir par ses conseils dans des domaines parfois éloignés de son subiectum ${ }^{2}$ propre et spécifique. Il s'agit de domaines éloignés, mais seulement en apparence, étant donné que le subiectum du savoir médical - le corps humain en tant qu'il peut être guéri-, quoiqu'unitaire, est inséré de fait dans un ensemble de relations et de conditionnements qui l'influencent, parmi lesquels, avant tout, le lien entre l'âme et le corps et les rapports avec le milieu environnant. Pour cette raison, souvent le médecin se présente presque comme l'incarnation d'une encyclopédie spécialisée ${ }^{3}$. Autour du noyau constitué par la doctrine médicale proprement dite, doivent en effet s'agréger des compétences variées, plus spécialisées et subsidiaires (dites d'ailleurs subservientes). Outre les arts libéraux, évidemment, toujours définis comme nécessaires depuis les Étymologies d'Isidore de Séville ${ }^{4}-$, le médecin ne peut ignorer l'astrologie, la physiognomonie, l'alchimie, l'oniromancie, ni l'art culinaire. Il ne peut ignorer non plus les aspects et les remèdes de l'activité thérapeutique " populaire ", qu'il accepte ou qu'il rejette, mais qu'il ne peut négliger, et qui confinent parfois à la magie. Surtout, étant donné l'importance des « accidents de l'âme " et de la confidentia que le praticien doit susciter chez le patient, étant donné aussi le rôle que joue l'imaginatio dans la thérapie, le médecin devra développer et mettre en pratique une sensibilité psychologique fine, fondée certes sur les doctrines médicales, mais aussi sur une fréquentation quotidienne des patients. 
2 C'est cette compétence multiforme, unifiée toutefois parce que tendant vers une même finalité, le bien-être d'un unique subiectum, qui rend le professionnel apte à fournir des conseils sur des sujets très variés ${ }^{5}$. Il s'agit souvent de suggestions qui concernent aussi des domaines psychologiques et des attitudes morales. Lorsque, ensuite, ces conseils se structurent dans le schéma formel des Regimina ${ }^{6}$, il n'est pas rare que les prescriptions du médecin revêtent une tonalité et une finalité proprement morales: de fait, les regimina contiennent des directives relatives à l'usage correct des six res non naturales ${ }^{7}$; ces dernières sont par ailleurs les seules choses sur lesquelles le sujet peut exercer un choix véritable, donc une délibération éthique. Au regard de ces res, le médecin est donc également guide et maître, il doit et peut exhorter, réprimander, inciter à un changement, avec comme objectifs des valeurs comme la tempérance, la prudence, l'équilibre qui contribuent à restructurer le style de vie global du destinataire, lequel n'est pas nécessairement malade.

3 Cette compétence et la capacité qui en résulte de «donner des conseils " (fondée sur l'autorité doctrinale mais aussi morale du médecin ${ }^{8}$ ) le rendent en outre apte à être l'interlocuteur indispensable d'autres experti et probi viri-juges, fonctionnaires urbains, prêtres, inquisiteurs - qui le consultent pour délibérer avec compétence et rectitude dans des situations complexes réclamant des approches multiples: la réglementation des mesures sanitaires pour la ville" ${ }^{9}$ l'exercice de la justice avec les expertises judiciaires ${ }^{10}$ et l'établissement des causes de la mort lors des procès ${ }^{11}$, les procédures de canonisation ${ }^{12}$ et les visites pastorales ${ }^{13}$. Enfin, peut-être la manifestation la plus éclatante de cet assemblage de compétences variées que le médecin peut unifier avec sa doctrine, et de valeurs, y compris morales, que son savoir peut véhiculer estelle constituée par la figure du médecin de cour ; cette figure est, en un sens, nouvelle dans le contexte particulier de la société seigneuriale italienne ${ }^{14}$. Les « conseils » et les interventions du médecin-conseiller du seigneur au $\mathrm{Xv}^{\mathrm{e}}$ siècle peuvent toucher - outre les champs normaux de la diététique et de la thérapie - des domaines politicodiplomatiques, même si c'est de manière fortuite. Il est toutefois significatif qu'ils se traduisent souvent en directives pédagogiques et éthiques, jusqu'à rejoindre les avertissements religieux destinés au seigneur ou à toute sa cour ${ }^{15}$.

Les conseils du médecin : règles de comportement

4 C'est durant cette évolution et surtout par ce dense réseau de conseils, demandés, écrits et échangés avec d'autres praticiens et experts, que s'étend progressivement le rayon d'influence du médecin savant. Mais il faut surtout souligner que la capacité même de «donner des conseils»-qui semble consubstantielle aux fonctions du médecin ${ }^{16}-$, réclame à son tour orientation et conseil ${ }^{17}$. En effet, les médecins, euxmêmes producteurs et destinataires de conseils «techniques » (de arte), reçoivent des directives d'autres ou s'auto-prescrivent - dans le circuit de leur propre communauté scientifico-professionnelle - des conseils et des suggestions de morale et de comportement (relatifs, entre autres, précisément à l'art de donner des conseils).

Dans le premier cas, il s'agit de règles dictées par la pastorale ou de strictes normes conciliaires qui concernent le bon exercice chrétien de la profession médicale (le général des dominicains Humbert de Romans et le médecin Nicolò Falcucci les qualifient de Mandata Ecclesiae, et Henri de Mondeville de documenta fidei catholica $\left.{ }^{18}\right)$. Ce sont des directives que les médecins peuvent parfois avoir des difficultés à accepter et qui peuvent faire l'objet de (très) prudentes discussions ${ }^{19}$. Ce sont toutefois des règles normatives, parfois même judiriquement, et elles ont comme objet principal la 
sauvegarde du salut de l'âme du médecin et du patient au cours de leur relation, ainsi que la réglementation des prérogatives respectives du médecin et du prêtre à l'égard du malade. Dans le deuxième cas, en revanche, nous sommes face, non à des préceptes, mais précisément à des conseils, que Nicolò Falcucci appelle monimenta ${ }^{20}$. De telles suggestions visent seulement à orienter, mais elles apparaissent contraignantes parce qu'elles sont (ou devraient être) partagées et décidées par la communauté des praticiens, et parce qu'elles tirent leur origine des auctoritates les plus reconnues de la médecine pratique et professionnelle. Elles se situent dans une zone mouvante, qui n'est pas connotée de manière décisive et univoque, et qui se place entre les lois et les obligations d'une part - judiriquement sanctionnées par les statuts des corporations et des Collegia medicorum - et les prescriptions d'autre part - juridiquement tout aussi contraignantes - du droit canonique sur la profession médicale ${ }^{21}$.

6 Il s'agit du domaine du comportement, un domaine très délicat, car lié aux multiples rapports quotidiens du médecin : avec le patient, avec ses familiers, avec ses collègues, avec les assistants et les concurrents de divers types. Ce domaine, remarquons-le, ne coïncide ni avec les normes laïques ni avec les normes religieuses. Il s'agit donc d'un contexte de relations où les perspectives proprement morales, les exigences professionnelles, les règles d'un comportement éduqué interfèrent avec des phases plus spécifiquement techniques - visite, diagnostic, prognostic, thérapie -, qu'elles peuvent soit compromettre, soit favoriser. Ici le médecin doit sauvegarder sa bonne réputation, sa renommée dans la ville, mais il doit aussi promouvoir des rapports corrects et affables avec chaque malade, sa famille et son entourage. Il ne peut négliger les appels de sa conscience chrétienne, ni sous-évaluer les embûches d'une pratique professionnelle très compétitive, continuellement menacée par de possibles fraudes (celles des pharmaciens, des astantes), par la médisance du «peuple», par la susceptibilité et les tromperies de collègues désireux de «voler» des malades. Dans ce réseau très complexe de rapports, enfin, le professionnel doit faire, à son tour, des choix dont certains demeurent facultatifs, au sens où ils ne sont pas seulement de type technique et thérapeutique; les auctores le guident, certes, mais dans chaque phase des nombreuses relations qu'il noue, il peut être amené à devoir prendre des décisions rapides et inattendues à propos de son propre comportement. Il n'est pas étonnant que ce niveau de professionnalisme soit continuellement "enseigné » dans des traités spéciaux et ou dans des sections de textes ${ }^{22}$, et qu'il soit même parfois « mis à jour » au regard des sages conseils fournis par les auctores.

Henri de Mondeville : instructions aux chirurgiens

7 Instruire ses confrères, c'est ce que déclare vouloir faire Henri de Mondeville dans de nombreux notabilia preambula et contingentia de sa Chirurgia ${ }^{23}$. Maintes fois, il rappelle que, dans la situation actuelle où des opérateurs variés - médecins, chirurgiens litterati mais aussi rustici, charlatans divers (qu'il décrit à l'évidence à partir d'une expérience personnelle) - présentent des prétentions thérapeutiques dans un "marché de la santé » apparemment surpeuplé et compétitif, il est nécessaire que le vrai chirurgien dispose non seulement de la doctrina relative aux interventions spécifiques de son artscience, mais aussi d'une authentique et propre doctrine, bien reconnaissable, sur le comportement professionnel, laquelle ne doit plus être confiée au simple usage et au bon sens, ou aux indications - parfois contradictoires et souvent éparses - des auteurs : "On voit, d'après ce qui vient d'être dit, qu'un chirurgien franc et consciencieux a souvent à souffrir de sa franchise et de son honnêteté, et qu'un chapitre sur la doctrine lui est nécessaire, afin de résister aux fourberies des chirurgiens et des médecins 
trompeurs, et à celles des malades, dont il est difficile de se garder $»^{24}$. Il semble presque que, dans ces amples sections du texte, se concentre la vaste gamme des rapports humains (affectifs, "passionnels ", rapports de dépendance et même rapports éthiques) en jeu dans la profession, mais programmatiquement exclus du regard neutre et objectivant du médecin. Celui-ci considère les seuls aspects «techniques" (symptômes, signes, degrés de douleur, forme des blessures) - et c'est justement pour cela qu'il peut élaborer une approche scientifique à l'égard de celui qui ne devient patient que de cette manière ${ }^{25}$. Ici, il semble presque que cette gamme de rapports se mêle à ces directives et ces conseils, qui sont désormais eux-mêmes traduits en doctrine autant que possible. Et de fait, Henri souligne qu'ils n'ont jamais été mis par écrit ${ }^{26}$ de manière si analytique et systématique; ce sont cependant des instructions essentielles pour définir la profession et ses fins : la guérison et sa rétribution ${ }^{27}$.

8 Si donc le médecin doit avoir deux yeux vigilants - comme le soutient Galien - attentifs à ces aspects, Henri de Mondeville estime que, pour « avoir à l'œil » toute les relations professionnelles et leurs facettes insidieuses, l'art chirurgical doit être doté d'au moins cinq $y^{2} \mathrm{x}^{28}$ : il n'en faut pas moins pour contrôler toutes les situations délicates auxquelles le chirurgien peut se heurter. On ne doit pas croire, par ailleurs, souligne-til, qu'avec ces conseils et instructions, il entende «enseigner à faire le mal, mais à connaître le mal pour l'éviter... et si certains conçoivent le mal à partir de ce qui vient d'être dit, ce n'est pas la vérité du discours qui sera en cause, mais la méchanceté de celui qui le comprend » : ses conseils, donc, ne seront pas de « mauvais conseils ${ }^{29}$.

Le rapport avec le patient est indubitablement central mais, dans sa Chirurgia comme dans d'autres textes plus ou moins contemporains, il me semble que les relations avec les collègues, ainsi que l'éthique et l'étiquette des consultations, sont tout aussi intéressantes. En fait, ces relations sont peut-être les plus liées à l'évolution sociale de la profession et elles sont révélatrices des rapports complexes entre opérateurs divers. Nous pouvons ainsi ranger les conseils-instructions d'Henri de Mondeville selon les différents niveaux d'articulation et de complexité des situations qui viennent d'être évoquées.

Le chirurgien et ses collègues

10 En premier lieu, il est chaudement recommandé au médecin de disposer d'un socius fidèle (ce peut être un disciple en formation, voire un collègue à peine formé), auquel il peut demander un consilium en pleine confiance ${ }^{30}$. Outre ce rôle essentiel, le socius/ famulus a les tâches délicates d'un "procurateur " ${ }^{31}$ : c'est à lui de s'informer préalablement des conditions cliniques et économiques du patient, de solliciter avec politesse les échéances et les types de paiement; il pourra aussi remplir les fonctions de son supérieur lors de certaines visites; naturellement, il ne devra jamais le remplacer entièrement ${ }^{32}$. Son manuel se voulant le plus complet possible, Henri se montre aussi très sensible aux difficultés que rencontre inévitablement le jeune chirurgien (cyrugicus novus), auquel il n'épargne pas les conseils, dictés aussi par sa propre expérience ${ }^{33}$, par exemple sur la manière de se débrouiller avec les patients anxieux et surtout avec les collègues plus connus ${ }^{34}$. Il est aussi dangereux de se montrer trop plein de compassion à propos des souffrances du malade ; les patients, et plus encore les vulgares illitterati, ne placeront pas en lui l'indispensable confiance et se moqueront de lui ; il sera donc utile pour ce débutant, plus que pour les chirurgiens affirmés, de prescrire des potions (qu'il sait être inutiles) propter defensionem contra vulgus, qui, de toute façon, réclament un remède, même s'il n'en a que l'apparence. Le délicat domaine des rapports entre 
médecins et chirurgiens - comme on peut le prévoir - rend nécessaires des conseils nombreux et détaillés. Certes, la meilleure situation à espérer est celle dans laquelle entrent en relations des professionnels scientifici et experti : «c'est une consolation de se rencontrer partout avec de telles gens, parce qu'ils approuvent les expériences des hommes probes et experts, suppléent aux ignorances et aux erreurs de ceux qui sont moins expérimentés, et les corrigent courtoisement et sans rien dire $»^{35}$. Mais une telle collaboration respectueuse et fructueuse semble bien rare au regard des stratégies de dénigrement et de tromperies réciproques qu'Henri décrit en détail à de nombreuses reprises $^{36}$. Il sera donc bon de fournir des conseils prudents au chirurgien. Tout litteratus qu'il est, le chirurgien par exemple ne doit pas se mêler de prescriptions de laxatifs forts " pour ne pas encourir la malveillance et l'indignation des médecins » (ne incurrat indignationem medicorum et malevolentiam) ${ }^{37}$. Du reste, en général, de même qu'il est nécessaire que les frères et membres d'une même famille aient une idée claire de leurs biens respectifs, de même il sera prudent de définir les domaines propres (certae meta $^{38}$ ) du médecin et du chirurgien, même si, dans le quotidien de la profession, malheureusement, de telles distinctions ne peuvent être aussi nettes et rigides. Par exemple (et c'est un indice intéressant de la hiérarchie professionnelle qui est en train de se former, des rapports conflictuels et des contrôles réciproques exercés entre opérateurs de divers rangs), la phlébotomie ${ }^{39}$ est à la fois un instrumentum medicorum et un opus chirurgicum, "un moyen employé par les médecins et une opération chirurgicale » : à qui incombe-t-il donc de l'effectuer? Au chirurgien sous la direction du médecin? Au médecin à condition qu'il soit aussi chirurgien ? En réalité, la pratique professionnelle effective, au cours du temps, semble avoir résolu la question (bien que ce soit sous une forme qui n'est peut-être pas pleinement appropriée). En effet, «les médecins ont depuis longtemps abandonné cette pratique aux chirurgiens, parce qu'elle est indigne d'eux, disent-ils, et peut-être pour un autre motif. Plus tard, les chirurgiens ont laissé cette opération aux barbiers pour deux raisons : 1. parce qu'elle rapporte peu ; 2. parce qu'elle est facile à maîtriser et de peu d'importance $»^{40}$.

11 Tout aussi délicats, les rapports avec les chirurgiens rustici et orgueilleux, quoique illiterati, sont peut-être aussi plus difficiles à contrôler, mais ils sont abordés avec des instructions plus draconiennes ${ }^{41}$. Du reste, les illiterati ne sont-ils pas la cible polémique de l'ensemble du texte d'Henri de Mondeville? La coordination correcte (objet de l'un des principaux Mandata Ecclesiae) entre chirurgien et prêtre semble en revanche désormais acceptée sans trop de difficultés : comme Henri le répète de nombreuses fois sans commentaire, le patient se sera déjà adressé au prêtre quand le chirurgien commencera à opérer, de même qu'il se sera déjà occupé de son propre testament. Voilà pour le comportement public. Mais quelle est l'importance respective des deux figures, le prêtre et le médecin? Henri, en faisant la liste des motifs que le chirurgien doit signaler au client-patient pour être récompensé justement, donc dans la phase préliminaire de négociation de leur rapport, soutient que la chirurgie est plus noble que la médecine. Or, en s'appuyant entre autres sur les « interventions » chirurgicales de Dieu et du Christ ${ }^{42}$, il souligne que le confesseur «sauve seulement l'âme du fidèle (salvat solam animam confitentis), tandis que l'action du chirurgien ou du médecin a des implications plus étendues: ceux-ci sauvent les organes essentiels sans lesquels le patient ne pourrait vivre ou, pire, sans lesquels il ne serait pas en mesure de gagner de quoi vivre pour lui et les siens, et des familles entières iraient ainsi à la ruine. Mais il existe des cas d'urgence, des blessures avec d'importantes hémorragies pour lesquelles il convient d'intervenir rapidement. Et c'est ici que surgit le doute : qui doit agir le 
premier, le chirurgien ou le prêtre ${ }^{43}$ ? Henri (qui avance tout à la fois avec clarté et habileté rhétorique) déclare la question futile ; parce que, s'il est vrai que l'art est une chose, la foi en est une autre, et que s'il est parfois possible (mais difficile) que le prêtre et le chirurgien agissent simultanément, il n'en reste pas moins qu'il existe une loi précise qui règle les préséances et à laquelle on doit se tenir.

La consultation

Comme on pourra le noter, l'habileté et la doctrine du chirurgien sont essentielles dans tous ces rapports : il doit être sûr et conscient de ce qu'il fait, c'est-à-dire solide dans sa scientia et par conséquent également certain de la salva conscentia et du salut de son âme $\mathrm{e}^{44}$. Mais la préoccupation majoritairement mise en évidence (outre celle qui regarde la rétribution) concerne le renom, la renommée et la réputation, liés trop souvent à l'opinio et sententia populi ${ }^{45}$. Henri admet que cette attention se répand et traverse tous les contingentia et c'est le plus important; on devra dans tous les cas éviter que «s'élève contre nous un murmure dans le peuple».

13 La situation très délicate dans laquelle la consultation est nécessaire constitue une occasion de possible murmuratio négative, une circonstance dans laquelle tous ces problèmes de rapports apparaissent évidents et amplifiés. Il s'agit ici, il faut le remarquer, de donner des conseils sur l'art de formuler des consilia, c'est-à-dire à la fois des avis et des consultations. Que cela représente un moment de toute façon difficile de la pratique professionnelle, c'est ce dont témoignent le fait qu'Henri l'aborde de nombreuses fois avant de l'affronter directement et explicitement, que toute la foule des auctoritates (qu'elles soient spécifiquement déontologiques ou plus générales) soit mobilisée, et qu'enfin, la question que ces mêmes autorités ne résolvent pas soit proprement le premier problème qui se pose dans ce contexte, à savoir : vaut-il mieux se fier à un seul professionnel fiable ou à plusieurs opérateurs?

Plus d'un médecin - parmi lesquels des contemporains ou des maitres de Mondeville sur la base des mêmes auteurs que lui, se sont posé ce problème classique, qui investit aussi bien les domaines théoriques (les éventuelles meilleures possibilités de diagnostic) que les comportements professionnels (les rapports plus complexes avec les collègues). Pietro d'Abano y consacre la Diffentia VII ${ }^{46}$ du Conciliator; il recourt également à des textes éthiques et politiques d'Aristote, au commentaire à l'Éthique d'Eustrate, à Proclus, au De causis, outre Galien, Damascène et Celse. Pietro se prononce, évidemment, pour une viam mediam : que le malade se fie donc à plusieurs médecins, si nécessaire, mais il faut que l'un d'entre eux ait la prééminence, comme du reste cela arrive dans le régime politique (le corps du patient est comparé ici à une civitas bene recta). Mieux vaut que ce protomedicus soit le plus diligent, le plus renommé et le plus aimé du patient. Il lui incombera ensuite de choisir les autres médecins parmi ses socii probiores ou parmi ses disciples (trois au maximum) et de recueillir leur divers motiva et consilia, en en coordonnant la pluralité : la pluralité est en fait cause d'erreur «quand elle n'a pas été ramenée à l'unité ». Pietro, par ailleurs, n'explique pas comment - sinon par l'autorité du protomedicus - on atteint concrètement cette concorde souhaitée.

15 Arnaud de Villeneuve ${ }^{47}$ aborde le sujet dans un discours plus global dédié à l'écriture des aphorismes et au tableau de faits qui permettent au médecin d'ordonner par écrit les conditions variées et successives de chaque patient et les caractères de sa maladie après la première visite et au cours des autres, tableau grâce auquel il parvient au diagnostic. Ces notes ordonnées (qu'il porte toujours avec lui durant ses visites) lui seront ensuite très utiles quand - si des incertitudes demeurent - le médecin soignant 
devra s'entretenir avec un savant confrère, ou jugera utile de convoquer une vraie conférence, appelant aussi des médecins exerçant au loin. Arnaud explique aussi, ou mieux, il raconte ${ }^{48}$ - pour une plus grande clarté didactique - différents cas/exempla ${ }^{49}$ de deux médecins qui soignent ensemble, mais ne tiennent pas compte de leurs consilia respectifs, pour le préjudice assuré du malade. Les consilia/aphorismes et les cas/ exempla qu'Arnaud propose ici peuvent être considérés comme un modèle assez intéressant d'une autre voie possible d'écriture médicale - et sans développement ultérieur - par rapport au parcours que les consilia écrits emprunteront en Italie ${ }^{50}$; ils signalent certainement aussi une grande différence d'organisation autour de l'écriture médicale entre professionnels français et italiens, et entre les écoles de Montpellier et celles de Bologne et Padoue; mais même ici, il est dit peu de choses à propos de la situation concrète (effective et non ficta), où plusieurs médecins se trouvent confrontés au chevet du malade ${ }^{51}$.

Les instructions du médecin Alberto de Zancari semblent un peu plus concrètes que celles du théologien-moraliste, auteur anonyme du Decem quaestiones de medicorum statu. Le premier introduit une distinction évidente, mais toutefois utile: si le praticien associé à la cure est un plebeius, «il ne faut pas parler avec lui » (cum illo non conferas), afin de ne pas lui donner un rôle et un crédit qu'il ne mérite pas; si en revanche le collègue est doté de scientia doctrinali, il devra décider avec lui de la manière d'agir. Mais il faudra toutefois faire en sorte que le consilium du premier médecin ait force d'obligation pour les assistants/astantes, ce qui s'obtient en étant particulièrement diligent lors des visites. Le texte pastoral, de son côté, dédie une quaestio ${ }^{52}$ entière au problème de la plus grande utilité de la présence d'un ou plusieurs médecins. Ici, l'unique auctoritas invoquée est Aristote, mais aussi l'« Aristote » du Secretum secretorum où, en effet, le thème de la pluralité des médecins est traité ${ }^{53}$. La quaestio est résolue en faveur de plusieurs médecins par consultation; parce qu'ainsi, ils ont plus d'expérience, et parce que le malade de haut rang est mieux protégé. Mais si l'entretien à plusieurs garantit que - dans une science tournée vers le particulier comme la médecine - l'on puisse parvenir à une plus grande certitude du raisonnement au sujet des experimenta (faits expérimentaux et recettes) ici essentiels, qu'on se souvienne aussi, avertit l'auteur (retournant ainsi aux préoccupations de Pietro d'Abano) qu'« une pluralité de médecins ne doit pas conduire à la nouveauté, mais à l'unité ", et que la collacio doit se résoudre en concorde. On peut encore une fois se demander comment.

La collatio selon Henri de Mondeville

Indubitablement, Henri de Mondeville fournit le manuel le plus concret et le plus détaillé, riche de directives pratiques à suivre effectivement durant la consultation. Comme cela a été dit, il fait allusion à de nombreuses reprises au thème dans divers autres notabilia. L'argument apparaît évidemment quand il aborde les rapports entre médecins et chirurgiens ${ }^{54}$; mais plus intéressante et plus pertinente est l'allusion (quand il enseigne à choisir un cyrurgicum sufficientem et aborde la question « un ou plusieurs chirurgiens? $\aleph^{55}$ ) qui est faite aux sectes des chirurgiens (sujet dont Henri traite à de nombreuses reprises et avec différents desseins dans la Cyrurgia). Il semble donc qu'à Paris, il arrive que les patients appellent au cours de leurs traitements une infinité de médecins de différentes sectes, pour qu'ils tiennent des conférences. Henri prévient: dans ce cas, "plus on est, moins on se sent responsable »; et si quelqu'un connaît " quelque chose de facile et d'éprouvé », « il ne le révèlera jamais au plus grand nombre ». C'est ainsi que se produit la situation dans laquelle on risque le plus de se 
fourvoyer du point de vue médical - risque d'ordre épistémologique plus encore que pratique. Mieux vaut donc que soient convoqués deux ou trois opérateurs et qu'ensuite il n'en reste qu'un qui ait littéralement "exprimé » des autres tout ce qui peut être utile. Mieux vaut encore (autre allusion ${ }^{56}$ à la préférence pour un seul responsable), dans la mesure où la multitude engendre la confusion (puisque l'on fait en vain à plusieurs ce que l'on peut faire à très peu), que l'on choisisse dès le début un seul cyrurgicum expertum et fidelem; si cela est vraiment nécessaire, qu'ils soient au maximum deux (comme, du reste, le voulait Arnaud de Villeneuve) et "qu'ils soient amis, de la même secte, unis de cœur et d'âme "; s'il en survient un troisième, que celui-ci vienne seulement pour discuter et "apporter la concorde entre eux ». Ce qui est donc à nouveau proposé comme préférable (du point de vue du choix du patient, cette fois), c'est le couple formé du « chirurgien accompagné par un socius» qui avait déjà été conseillé au professionnel pour une pratique efficace.

Enfin, dans le $22^{\mathrm{e}}$ notabile introductorium ${ }^{57}$, Henri se prépare à traiter de la véritable collatio; l'examen attentif comprend des instructions, des renvois à des directives fournies dans d'autres sections, l'illustration d'une possible quaestio interne entre les participants ; cela se termine - pour confirmer la finalité didactique et pratique de ces instructions - par un exemplum sensibile cyrurgicum, ut melius videatur. Les instructions sur la procédure de la collatio - qui survient quand plusieurs médecins sont appelés ensemble - sont énumérées dans l'ordre. En premier lieu, ces participants peuvent tous être des médecins, des chirurgiens ou un groupe mixte; il faut espérer qu'ils ne soient pas envieux, difficiles ou insolents; il n'y a qu'ainsi qu'ils peuvent utilement s'entretenir. Tout d'abord après s'être informés de la maladie en cours, tous, l'un après l'autre, doivent voir et toucher le malade, chacun notant les signa et particulares considerationes. Puis, que celui qui jouit de la plus grande autorité (autenticus) - mieux vaut qu'il soit médecin - s'adresse au malade et, qu'au nom de tous, il l'interroge sur les circonstances de la maladie (débuts, symptômes précédents, questions formant une séquence cohérente comme cela est prévu dans le $45^{\mathrm{e}}$ contingens). Ensuite, ils sortiront tous de la chambre du malade, pour entrer dans une pièce où ils seront seuls, et ce sera alors la phase plus délicate de leur entrevue. En effet, «dans tout entretien, que les maîtres disputent entre eux pour mieux discuter la vérité »; or, souvent, dans la fougue du débat, ils s'expriment d'une manière qui pourrait faire supposer (ce qui est parfois le cas) le début d'une dispute et d'une discorde ${ }^{58}$. À cet égard justement, à nouveau, le plus âgé ou le plus connu et le plus éminent donnera à tous la parole : d'abord aux plus jeunes et aux moins connus, parce que si ces derniers parlaient selon l'ordre hiérarchique en dernier, jamais ils n'oseraient dire quelque chose de différent des maitres renommés qui les ont précédés, et alors ce serait comme s'il n'y avait pas eu de collatio. Le magister doté d'autorité demandera alors avec ordre à tous ses confrères quelle est la maladie, de quel nom elle est désignée par les experts, quelles autoritésen parlent et dans quels textes. Enfin, il demandera (voici la quaestio «interne»), si la maladie ainsi définie est curable et de quelle manière.

La collatio et la pratique médicale

19 Différents aspects des instructions données à propos de la collatio par Henri - ce sont ses « conseils à propos de la méthode à suivre pour donner des conseils » - se révèlent neufs, jusque dans leur détail peut-être, par rapport à d'autres textes. Mais, avant tout, soulignons que la tradition française est confirmée ${ }^{59}$ : la consultation doit et ne peut survenir qu'en présence du malade, dans la pièce tout près de son lit. Elle ne doit pas 
subir ces transformations épistémologiques et d'écriture causées par «l'éloignement du chevet ", qui permettent aux médecins italiens d'écrire des consilia in absentia (qui, précisément, sont techniquement des consultations, des échanges d'avis entre les médecins, mais qui sont établis par écrit et loin du patient, ce qui souvent les transforme en petits traités doctrinaux ${ }^{60}$ ). Le consilium in absentia (notabile introductorium $\mathrm{n}^{\circ}$ 26) est donc exclu, même si, comme l'écrit Mondeville, "à nous, les chirurgiens, on demande très souvent un conseil sur le traitement de maladies que nous n'avons pas observées, et ne pouvons pas voir à cause de l'éloignement des patients ». Il n'est pas permis de fournir semblable consilium parce qu'il ne respecterait pas (à la différence de la collatio) les règles de arte (qui imposent de voir, toucher, questionner) et pour cette raison ne garantirait pas la salva conscientia du professionnel. En outre, le consilium in absentia ne peut tenir compte des variations continues, du processus de la maladie - processus sur lequel Henri insiste souvent dans d'autres passages, y compris en des termes diagnostico-thérapeutiques -, qui a ses temps et son développement. Aucun nuncius ou lettre ne peut remplacer la main, les yeux, les questions de l'opérateur expert, ni ne sait rapporter ce qui est arrivé entre-temps : pour ces raisons, un tel consilium «n'est pas sûr et ne fait pas de bien ».

De fait, ce n'est pas un hasard si les consilia italiens écrits (in absentia) ont surtout pour objet les affections chroniques et à évolution lente, qui n'ont rien à voir avec les situations urgentes et rapidement changeantes qui d'habitude nécessitent l'intervention du chirurgien. Ainsi, le chirurgien de confiance ne doit pas donner de consultation en l'absence du malade : du moins, il ne fournira pas un consilium curatif mais seulement palliativum. Cela correspond en fait aux rares consilia écrits in absentia dans l'aire française ${ }^{61}$, à l'image de celui des quatre célèbres médecins de Montpellier (parmi lesquels Giordano de Turre et Gérard de Solo) qui expriment leur avis sur la paralysie qui menace un patient illustre: certes, ils écrivent leurs instructions in absentia (du moins, à ce qu'il semble), mais leur consilium consiste essentiellement en un regimen diététique : et, comme le dirait Henri, «il peut tout au plus faire du bien et ne nuit pas».

21 Quelques documents du début du $\mathrm{Xv}^{\mathrm{e}}$ siècle permettent de recueillir la crédibilité historique de la description et prescription d'Henri au sujet de la collatio. Mais ils permettent aussi de cerner la distinction - qui ne parait pas aussi nette - non pas tant entre les consilia (qui sont de fait diffusés et structurés différemment en France et en Italie) qu'entre les usages professionnels italiens et français. À propos du premier point, les consignations écrites des consultations, conseils et recettes du maître Guillaume Boucher ${ }^{62}$ et de ses collègues revêtent un intérêt particulier. Ils sont assurément des documents de la vie professionnelle, transcrits par un socius-élève (un certain magister de Almania) qui fréquentait leur communauté professionnelle et qui, probablement, a voulu se constituter un aide-mémoire utile pour sa propre pratique. Ces secreta et consilia semblent, à presqu'un siècle de distance, l'enregistrement sur le vif de ce qu'Henri a décrit: à de nombreuses reprises, en fait, il est fait référence aux délibérations et au commun accord des maîtres. On y trouve souvent rapportés le type et l'enchaînement des questions faites au patient et les gestes d'exploration des médecins. Un chirurgien habile et une sage-femme sont même convoqués pour une consultation. Dans trois cas au moins ${ }^{63}$, on peut déduire la présence de plusieurs médecins pour une consultation dans la chambre du patient. Pour une commodité d'enregistrement (par laquelle sont dressées les listes des conclusions auxquelles ils 
arrivent) ou parce que, de fait, la méthode de la collatio fonctionne, ces médecins apparaissent tous d'accord, surtout pour ce qui concerne les prescriptions opératives, résultat de la consultation, même si, dans un cas, sont rapportées différentes interventions des participants $\left(n^{\circ} 87\right)$. En ressort, évidemment, l'autorité de Boucher ; elle est soulignée et les nombreuses visites qu'il effectue, lui et ses compagnons, pour suivre l'évolution, le processus d'un cas difficile, sont signalées ( $\mathrm{n}^{\circ} 88$ ).

Mais sur cet aspect particulier-les temps de la maladie, qui semblent laissés résolument dans l'ombre dans les consilia italiens canoniques plus $\operatorname{tardifs}^{64}-$, c'est précisément un document italien qui nous informe que les distinctions trop nettes indispensables pour définir les genres littéraires comme les consilia-, ne sont pas si efficaces pour délimiter les pratiques de la consultation dans un sens plus large. Dans une lettre de $1407^{65}$, le médecin Lorenzo Sassoli de Prato raconte qu'il a visité un patient deux fois et que ce dernier est en danger; il voudrait alors se concerter avec son collègue Cristofano (le premier médecin soignant), mais il ne parvient pas à se mettre en contact avec lui et ne dispose donc pas d'informations sur le cours antérieur de la maladie : inconvénient pénible, parce que « en un seul jour, la dispositio change ». En outre, sa conviction profonde est que « c'est une honte grave pour un médecin et un dommage pour l'âme d'ordonner ou de ne donner aucun remède s'il ne voit pas pourquoi il le donne». Les archives de cour qui concernent les maladies de patients illustres $\mathrm{au} \mathrm{Xv}^{\mathrm{e}}$ siècle sont tout aussi significatives de ce type de concorde entre des groupes de médecins (qui sont presque des collationes permanentes) et de l'attention plus que quotidienne portée jusqu'aux plus petits changements de situation pathologique ${ }^{6}$. Certes, de tels documents sont trop fortement conditionnés par les règles très spécifiques de la communication (et de l'assistance médicale) de cour pour témoigner d'une pratique commune; ils témoignent toutefois qu'il n'y a pas un seul mais différents modes de dare consilia et de se situer dans une collatio.

Enfin, le manuel de Nicolò Falcucci, dans les premières années du Xve siècle, permet de voir à quel point la pratique décrite par Mondeville est alors en passe d'être considérée comme une pratique professionnelle à conseiller. Dans son premier sermo - comprenant de très nombreux renvois aux auctores, comme tout manuel, et consacré à la définition de la médecine, aux figures, aux dons et aux devoirs du médecin-prévaut incontestablement, souvent à la lettre, la ligne définie par Pietro d'Abano sur de nombreux points. Falcucci conseille toutefois - comme Henri l'avait suggéré - que le médecin ait un idoneum discipulum qui l'assiste, que le professionnel consulte le médecin qui avait précédemment en cure le patient, que, en cas de nécessité - et bien qu'il vaille mieux se fier à un seul médecin, selon ce qu'affirme Damascène -, il accepte d'en consulter plusieurs et que ceux-ci soient « animés de bonnes intentions, dénués d'envie et de rivalité, et qu'ils ne cherchent pas la vaine gloire ». Celui que le malade apprécie le plus aura l'imperium dans une telle assemblée, mais il ordonnera un regimen approprié après avoir entendu l'avis de tous ses confrères ${ }^{67}$.

Collatio et doctrine

Mais analysons plus en détail la structure du consilium licite et correct, c'est-à-dire l'avis exprimé par plusieurs professionnels in presentia dans la collatio décrite par Mondeville. En premier lieu, si la figure d'autorité du médecin le plus éminent reste centrale - comme dans d'autres textes -, on comprend bien ici son rôle, qui n'est pas seulement gratifiant, honorifique et rhétorique, mais aussi fonctionnel : il est de son devoir de faire que tous s'expriment au mieux. Qu'il n'étouffe donc pas, par son 
autorité, le débat, mais plutôt, le promeuve et le réglemente. En outre, ce qui est souligné une fois de plus, c'est la fonction déterminante de la doctrina, d'un savoir ordonné et organisé (qui comprend des experientia, des auteurs, des textes, une capacité de débat) pour définir le vrai professionnel.

À ce propos, on notera que le déroulement de la collatio est très mimétique par rapport à la forme de l'enseignement universitaire. Le lexique utilisé le montre bien : magistri, disputatio, veritas, queritur utrum. En outre, les participants doivent définir la maladie, aussi bien dans le sens de " poser un diagnostic » que dans celui de «donner un nom approprié ». Que l'on se souvienne alors des listes de synonymes et des explications terminologiques que l'on trouve souvent dans les commentaires médicaux. Ensuite sont invoqués les auteurs et les textes afférents au cas. Enfin, une quaestio est posée. Ici, toutefois, le résultat d'une telle procédure (qui ressemble beaucoup à un examen du curriculum) n'est pas seulement un exercice de doctrine, mais doit déboucher sur une pratique et mener au choix rapide d'un traitement. Il est aussi évident que la décantation des aspects moins professionnels et plus « humains » des participants, les "vices » très souvent énumérés comme étant propres à la profession (vaine gloire, orgueil, superficialité prétentieuse, mépris pour les inférieurs, duperies, fraudes et surtout rivalités bagarreuses), s'obtient - elle est réalisée de fait par le groupe de consultants - précisément à travers la neutralisation de ces aspects, négatifs pour l'objectivité de l'habileté savante, qui rend tous les thérapeutes, quoique différents, égaux dans la contribution au savoir et dans la compétence qu'ils peuvent offrir.

La morale d'une communauté professionnelle

Les magistri deviennent donc respectueux l'un de l'autre dans la collatio, et collaborent pour parvenir à une fin utile à la connaissance et à la pratique, tel ce magister de Alamania, membre d'un consortium in practica, auquel il collabore activement et qui l'enrichit.

Michael McVaugh juge cependant non approprié «de penser qu'Arnaud et ses contemporains ont traité d'éthique». "Car l'éthique», explique-t-il, "me semble impliquer des valeurs désintéressées partagées par une communauté consciente d'ellemême. Or le comportement que j'ai décrit était loin d'être désintéressé: il était instrumental, dans la mesure où il tendait à affermir l'acceptation de la médecine académique dans les années où elle commençait tout juste elle-même à s'établir " ${ }^{68}$.

D'évidence, le jugement de M. McVaugh dépend de sa conception de la nature de l'« éthique ", mais il me semble toutefois qu'on peut le partager pour une bonne part, à condition de le corriger et nuancer. Avant tout, le fait que les médecins et chirurgiens "se pensent" comme une communauté - certes pas dans les formes évoluées et juridiquement stables que McVaugh évoque comme propres au $\mathrm{xv}^{\mathrm{e}}$ siècle, mais dès avant cette date-, résulte de nombreux facteurs ${ }^{69}$, entre autres du fait que c'est précisément au groupe de rationabiliter practicantes qu'est confiée la fonction décisive de valider des recettes et des procédures (qui circulent dans la communauté ${ }^{70}$ ), ce qui en fait ainsi non plus des empirica dus au hasard, mais des experimenta scientifiquement utilisables. Le renvoi fréquent aux socii, au "troupeau $»^{71}$ des vrais experts, va dans le même sens; des expressions, ensuite, comme communitas sapientium ${ }^{72}$ peuvent permettre de se référer à une communauté, peut-être informelle mais consciente, de professionnels regroupés autour de certaines valeurs. L'ensemble de normes qui sont élaborées par cette communauté ne me paraît être ni une simple "étiquette ", ni seulement instrumentale; il est vrai toutefois que ces normes se fondent sur, et qu'elles 
renforcent essentiellement la valeur du savoir doctrinal, magnifié depuis peu par les médecins et encore plus récemment convoité par les chirurgiens. Qu'enfin ces textes, spécialement pour ce qui regarde le consilium et la consultation, aillent dans la direction d'une éthique - au moins dans le sens où on l'entend dans les perspectives de la théologie et de la pastorale contemporaines -, est également vrai.

La réflexion théologico-pastorale sur l'éthique du conseil apparaît ainsi ample et articulée, tout particulièrement aux XIII ${ }^{\mathrm{e}}$ et $\mathrm{XIV}^{\mathrm{e}}$ siècles $^{73}$ : le débat se porte plusieurs fois sur des questions relatives à la nécessité, naturelle et morale, de conseiller, sur la nature essentiellement relationnelle, politique et opérative du consilium, sur les conditions, les facultés psychologiques et les vertus qu'il réclame et met en mouvement. Il ne manque, évidemment, ni la distinction entre consilium de compétence (de arte) et un vrai et propre conseil moral, ni une réflexion sur la moralité des compétences professionnelle elles-mêmes. Quoi qu'il en soit, l'éthique du consilium élaborée ici tourne autour, non de la définition de valeurs désintéressées, mais essentiellement de la vertu de prudence pour obtenir des fins opératoires. Ce n'est pas par hasard que la prudence est une vertu à forte tonalité cognitive, point de conjonction entre les vertus intellectuelles et morales. Or, rien qu'à propos du consilium de arte, les théologiens et les moralistes insistent à l'évidence sur le cas si particulier de la médecine, reconnaissant qu'ici, il n'est pas seulement question d'habileté technique, de règles opératoires orientées vers une fin, mais que ces règles, pour le médecin, sont chargées d'un poids éthique ; en effet, de telles règles sont en premier lieu le savoir qui, avant tout par obligation morale (selon les théologiens et les moralistes des Summae casuum) doit être pleinement dominé et continûment approfondi et cultivé par le médecin ${ }^{74}$, et la prudence, étant donné que le médecin intervient dans des situations incertaines, contingentes, particulières. Par conséquent, le péché spécifique prévu pour les médecins (voire inhérent à leurs consilia) est l'ignorance doctrinale accompagnée d'une superficialité imprudente.

Dans ce cadre théorique sur l'éthique du consilium, on peut admettre que le conseil du médecin se range en général dans le domaine de la "prudence imparfaite ", dans laquelle celui qui conseille donne la directive technique correcte, tout en étant luimême non pas vertueux mais seulement compétent. On se rappellera toutefois que le médecin vise non seulement au bon résultat technique, mais aussi, assurément avec ses consilia, au bon style de vie (y compris éthique) de qui se confie à lui, au bien de la cité, au développement harmonieux de la personnalité du seigneur ; il s'oriente également, à travers son art, vers des niveaux de religiosité personnelle et des objectifs de salut qui, déjà, déplacent ses fins dans le domaine gouverné par la "prudence parfaite». La dynamique, ensuite, de la collatio en particulier, avec les vertus complémentaires qui sont réclamées ou qui s'imposent aux participants (et que l'on a rencontrées plus haut) me semble s'ouvrir à des espaces relevant proprement de l'éthique «scolastique » de la prudence, en plus de l'éthique de la compétence et de l'étiquette professionnelle ; pour ne pas dire que, même quand la compétence technique (de arte) est garantie, la prudence dans le conseil est toujours nécessaire, comme le rappelle Alberto de Zancari : "Quand certains vont trouver le médecin, le consultent et lui demandent un conseil, le médecin doit considérer la condition du demandeur et l'objet du conseil. Si le patient est digne de foi, et demande un conseil à propos de quelque chose de licite, il faut le lui donner. Si c'est le contraire, il faut s'y refuser absolument $»^{75}$. 


\section{NOTES}

*.Je remercie de tout cœur Marilyn Nicoud et Laurence Moulinier pour leur patience et leur aide généreuse.

1. La mutation, y compris dans le domaine de la déontologie, est soulignée par

M. McVAuGH, « Bedside Manners in the Middle Ages », Bulletin of History of Medicine, 71, 1997, p. 201-33 (auquel je renvoie aussi pour la bibliographie antérieure sur l'histoire de la déontologie médicale médiévale). $\mathrm{Cf}$. aussi W. ScHNEIER, Medical Ethics in the Renaissance, Washington, 1995.

2. Cf. C. CRISCIANI, « Consilia, responsi, consulti : i pareri del medico tra insegnamento e professione ", dans Consilium. Teorie e pratiche del consigliare nella cultura medievale, C. CASAgrande, C. CRisciani, S. Vecchio dir., Florence, 2004, p. 259-279.

3. Voir par exemple PIETRO D'ABANO, Conciliator controversiarum quae inter philosophos et medicos versantur, Venise, 1565 (reprod. an. Padoue, 1985), Differentia V ; cf. aussi F. ALESSIO, «Filosofia e scienza. Pietro d'Abano », dans Storia della cultura veneta, II. Il Trecento, Vicence, 1976, p. 171-206 ; J. AGRIMI-C. CRISCIANI, Edocere medicos. Medicina scolastica nei secoli XIII-XV, Milan-Naples, 1988, Appendice.

4. Cf. Isidori Hispalensis Episcopi Etymologiarum sive Originum Libri XX, éd. W. M. LINDSAY, Oxford, 1962, I, IV, XIII.

5. La dépression et le moyen de vaincre le mal d'amour, les aptitudes à certaines études des jeunes gens, les précautions à prendre lors de voyages dans des terres lointaines et la préparation de campagnes militaires, les déplacements des malades vers des lieux plus agréables et la disposition de leur chambre, les conversations et les distractions adaptées aux convalescents de différents âges, ou encore les techniques pour une bonne conception et la naissance d'enfants vigoureux.

6. Cf. M. Nicoud, Aux origines d'une médecine préventive : les traités de diététique en Italie et en France (XIII ${ }^{e}-\mathrm{XV}^{\mathrm{e}}$ siècles), thèse de doctorat, École Pratique des Hautes Études, Paris, 1998 (à paraître) ; P. GIL SOTRES, « Les régimes de santé », dans Histoire de la pensée médicale en Occident, I, Antiquité et Moyen Âge, M. D. GRMEK dir., trad. fr. Paris, 1995, p. 257-281.

7. Cf. L. GARCÍA BALLESTER, « On the origins of "Six-non-natural-things" in Galen », dans Galen und das hellenistische Erbe, J. KolLESCH-D. NIKEL dir., Stuttgart, 1993, p. 105-115.

8. Accentué, en particulier, avec un ton intellectualisant, dans certaines orationes : voir infra.

9. $C f$., entre autres études, M. Nicoud, « Médecine et prévention de la santé à Milan à la fin du Moyen Âge ", Siècle, 14, 2001, p. 23-37 (et la bibliographie citée) ; R. PALMER, "Physicians and the State in post-medieval Italy ", dans The Town and the State Physicians in Europe from the Middle Ages to the Enlightenment, A. W. Russel dir., Wolfenbüttel, 1981, p. 391-401; J. AGRIMI, C. CRISCIANI, « Charité et assistance dans la civilisation chrétienne médiévale ", dans Histoire de la pensée médicale..., op. cit., p. 151-174.

10. Cf. J. AgRimi-C. CRISCIANI, Les « Consilia » médicaux, Turnhout, 1994, en particulier p. 34-36, 102-104 ; C. CRISCIANI, « Consilia, responsi, consulti », loc. cit. ; E. DALL'Osso, L'organizzazione medico-legale a Bologna e a Venezia nei secoli XII-XIV, Césène, 1956. 
11. Cf. J. ShATZMiLler, Médecine et justice en Provence médiévale. Documents de Manosque, 1262-1348, Aix-en-Provence, 1989 ; ID., « The jurisprudence of the dead body : medical practition at the service of civic and legal authorities ", Micrologus, 7, 1999, p. 223-230 (avec la bibliographie spécifique).

12. Cf. J. ZIEGLER, "Practitioners and Saints : Medical Men in Canonization Processes in the Thirteenth to Fifteenth Centuries », Social History of Medicine, 12, 1999, p. 191-225. 13. $C f$. J. PeRARNAU i ESPELT, « Activitats i formules supersticioses de guariciò a Catalunya en la primera meitat del segle XIV », Arxiu de Textos Catalans Antics, 1, 1982, p. 47-78.

14. Cf. T. PESENTI, « Medici di corte e di Università », Medicina nei secoli, 9/3, 1997, p. 391-401; C. CRISCIANI, « Tra Università, corte, città : note su alcuni medici "pavesi” del sec. XV », Annali di storia delle Università Italiane, 7, 2003, p. 55-70 ; EAD., « Michele Savonarola, medico : tra Università e corte, tra latino e volgare ", dans La filosofia in volgare nel Medioevo, N. BRAY-L. STURLESE dir., Louvain-la-Neuve, 2003, p. 433-449 ; I. NASO, Università e sapere medico nel Quattrocento. Pantaleone da Confienza, Cuneo-Vercelli, 2000 ; M. Rotzoll, Pierleone da Spoleto. Vita e opere di un medico del Rinascimento, Florence, 2000.

15. C'est le rôle de Michel Savonarole auprès des Este à Ferrare : $c f$. A. SAMARITANI, « Michele Savonarola riformatore cattolico alla corte estense a metà del sec. XV », Atti e memorie della deputazione provinciale ferrarese di storia patria, s. III, 22, 1976, p. 44-85.

16. En fait, quand on interdit aux charlatans de pratiquer, on leur interdit spécifiquement, entre autre, de consilium dare : $c f$. C. MALAGola, Statuti delle Università e dei Collegi dello Studio Bolognese, Bologne, 1888, p. 442. Vincent de Beauvais semble regrouper sous le terme de consilium l'activité médicale dans son ensemble : cf. Speculum naturale, Douai, 1624, XXXII, chap. XCIX, Qualiter consilio medici sit utendum.

17. La formulation de ces «métaconseils » (notamment ceux définis comme monimenta) semble ne pas faire référence (du moins explicitement) aux théorisations théologiques sur le consilium. Sur ce sujet, $c f$. C. CASAGRANDE, « Virtù della prudenza e dono del consiglio ", dans Consilium, loc. cit.

18. Cf. HenRi De Mondeville, Cyrurgia, éd. J. L. PAGel, Berlin, 1892, p. 96.

19. C'est le cas surtout de la question de la présence opportune du prêtre, qui pourrait impressionner le malade ; le problème, auquel sont également sensibles les théologiens, est toutefois résolu par la compréhension réciproque des exigences respectives : $c f$.

C. CRISCIANI, «Infirmitas, terapia spirituale e medicina », dans Storia della scienza Treccani, IV, Rome, 2001, spéc. p. 481-85.

20. Nicolò FAlCUCCI, Sermones medicinales, Pavie, 1481-1483, Sermo primo, fo $17 \mathrm{r}^{\circ} \mathrm{a}$ (numérotation moderne).

21. Cf. C. CRISCIANI, "Infirmitas », loc. cit. ; J. AGRIMI, « Le professioni mediche », ibid., p. 485-494.

22. Outre les « classiques ", parmi lesquels Johannes Mesue l'emporte avec ses Aforismi, voir le De instructione (adventu) medici attribué à ARCHIMATTEUS DE SALERNE (de nombreuses incertitudes demeurent à propos de ce texte), éd. S. DE RENZI, dans Collectio salernitana, Naples, 1852-1859, II, p. 74-75, V, p. 339-49 ; ARNAUD DE VILLENEUVE, De cautelis medicorum, dans Opera Omnia, Bâle, 1585, col. 1454-58 ; ALBERTO DE ZANCARI, De cautelis medicorum, éd. M. MORRIS, Leipzig, 1914 ; PIETRO D'ABANO, Conciliator, op. cit., Differentiae II et VII. 
23. Sur le texte et l'activité d'Henri, ainsi que sur la situation de la chirurgie, $c f$. M.C. Pouchelle, Corps et chirurgie à l'apogée du Moyen Âge, Paris, 1983 ; M. McVAUGH, « Royal surgeons and the value of medical learning : the Crown of Aragon ", dans L. GARCíA BALLESTER et al. (dir.), Practical Medicine from Salerno to Black Death, Cambridge, 1994 ; ID., "Stratégies thérapeutiques : la chirurgie ", dans Histoire de la pensée médicale..., op. cit., p. 239-255 ; ID., Introduction, dans GUY DE CHAULIAC, Inventarium sive Chirurgia magna, éd. M. McVAugh, Leyde, 1997 ; T. PesenTI, « Professores chirurgie, medici ciroici e barbitonsores a Padova nell'età di Leonardo Buffi da Bertipaglia († 1448) », Quaderni di Storia dell'Università di Padova, 11, 1978, p. 1-38; C. O'BoYLE, « Surgical texts and social contexts : physicians and surgeons in Paris, c. 1270 to 1440 », dans Practical Medicine, op. cit., p. 156-85 ; N. SIRAISI, « How to write a Latin book on surgery : organizing principles and authorial devices in Guglielmo da Saliceto and Dino del Garbo », ibid., p. 88-109; P. MURRAY JONES, « John of Arderne and the Mediterranean tradition of scholastic surgery », ibid., p. 289-321; J. AGRIMI-C. CRISCIANI, « The science and practice of medicine in the thirteenth century according to Guglielmo da Saliceto, Italian surgeon ", ibid., p. 60-87 ; D. JACQUART, La médecine médiévale dans le cadre parisien, Paris, 1998, spéc. chap. I, et passim. Cf. aussi, pour un point de vue particulier, C. CRISCIANI, « Artefici 'sensati : experientia e sensi in alchimia e chirurgia (secc. XIII-XIV) », dans Alchimia e medicina nel medioevo, C. CRISCIANI, A. PARAVICINI BAGLIANI dir., Florence, 2003, p. 135-159; M. McVAUGH, « Alchemy in the Chirurgia of Teodorico Borgognoni », ibid., p. 55-75.

24. $C f$. HENRI DE MONDEVILLE, Cyrurgia, op. cit., p. $72:$ « Ex praedictis patet quod cyrurgicus veridicus et fidelis propter veritatem et fidelitatem multotiens habet pati et quod sibi necessarium esset capitulum de doctrina, per quam posset resistere fraudibus cyrurgici et medici fraudulosi et etiam patientium, a quibus difficile est cavere ".

25. Sont encore utiles les considérations pionnières de J. REVEL-J.-P. PETER, « Le corps. L'homme malade et son histoire », dans Faire de l'histoire, J. LE GOFF, P. NoRA dir., Paris, 1974, III, p. 169-91.

26. HENRI DE MONDEVILle, op. cit., p. 75 ; p. 518 (« le chirurgien désireux d'exercer son art avec sagacité et habileté doit se reporter aux 25 notables préliminaires de toute la Chirurgie, aux 52 contigents relatifs au traitement des maladies chirurgicales, et aux 15 notables préliminaires spécialement consacrés au traitement des plaies. Il y trouvera un grand nombre de généralités utiles et qui d'ailleurs n'ont jamais été formulées dans les autres Pratiques de chirurgie ") ; à un certain point, Henri cède : trop nombreuses sont les circonstances à considérer, notamment dans le domaine astrologique et, par conséquent (p. 121), « qu'on laisse au talent et à l'ingéniosité naturels, ainsi qu'à l'art, à l'habileté et à l'expérience de l'opérateur, le soin de les déduire ».

27. Ibid., p. 75 (« de même que ce qui occupe tout entier le patient, c'est d'être guéri..., de même la préoccupation principale du chirurgien doit être d'être payé ») ; ailleurs (p. 131), Henri déclare que les connaissances du chirurgien seraient vaines « s'il n'avait l'art et la science de se faire payer, puisque c'est là son intention principale ».

28. Sur les « yeux » nécessaires à l'art chirurgical, cf. ibid., p. 75-76.

29. Sur ce thème, voir C. CASAGRANDE, Virtù della prudenza, loc. cit., et C. CRISCIANI, «Consilia », loc. cit., n. 68.

30. $C f$. HenRI DE MONDEVILle, Cyrurgia, op. cit., p. 76 (" il est nécessaire que le chirurgien ait... un associé légitime et fiable, dont il puisse prendre conseil et auquel il puisse recourir »).

31. Ibid., p. 74. 
32. Ibid., p. 74.

33. À ce sujet, $c f$. ibid., p. 142, 125, 126, 94.

34. "Valde periculosum cyrurgico non famoso operari aliter in aliquo proposito quam alii cyrurgici communiter operantur ». Bien que Mondeville soit particulièrement enclin à des innovations et qu'il souhaite le progrès continuel et inventif des procédures et des instruments des chirurgiens, il n'oublie pas les difficultés professionnelles d'un débutant.

35. HENRI DE MONDEVILLE, op. cit., p. 67 : « solatium est utique cum talibus operari quoniam proborum et expertorum experientiis acquiescunt et inexpertorum ignorantias et errores supplent et corrigunt curialiter et latenter ».

36. Ibid., p. 66 et passim.

37. Ibid., p. 95 ; aux médecins (p. 423) est également entièrement laissé le regimen ethicorum qui est pure medicinale.

38. Ibid., p. 334.

39. Ibid., p. 365.

40. Ibid. : « medici... ab antiquo operationem istam cyrurgicis reliquerunt propter indecentiam sicut dicunt, et forte aliud latet. Et ulterius istam operationem cyrurgici usque ad barberios repulerunt propter duo, 1) quia est operatio pauci lucri ;

2) quoniam ibi est magisterium paucum et leve... ». Cette hiérarchie est rappelée comme évidente dans les Decem quaestiones de medicorum statu (éd. R. PEITZ, Pattensen, 1978), pour évaluer les responsabilités respectives et également juridiques, en cas d'échec : p. $67:$ : Sed contingit quandoque quod ex minucione sangwinis, quam barbitonsor facit et medicus facit fieri, minutus debilitatur et moritur » (« mais il arrive parfois, à cause de la saignée que le médecin fait faire et que le barbier exécute, que le patient saigné s'affaiblisse et meure »).

41. HenRi De MONDEVILle, op. cit., par exemple p. 65, 67, 71, 118.

42. Ibid., p. 79-80, 133.

43. Ibid., p. 165.

44. Ibid., p. 134-35.

45. Cf. ibid., p. 99, contingens 27 ; cf. aussi p. 61 : « [chirurgicus] ad bonam famam quantumcumque potest, laboret ». Cf. PIETRO D'ABANO, Conciliator, op. cit., fo $5 \mathrm{r}^{\circ} \mathrm{bA}$ : « cum inde laudabilis consurgat fama »; ZANCARI, op. cit., p. $13:$ «Bonum commune civitatis zelet et patriam ; vulgi laudes non cupiat... Reverentias non spernat vulgarium ».

46. Cf. Conciliator, cit., fo $10 \mathrm{v}^{\mathrm{o}} \mathrm{aH}, 11 \mathrm{r}^{\mathrm{o}} \mathrm{aB}$.

47. Cf. ARNAUd DE VILLENEUVE, Explicatio super Canonem Vita brevis, dans Opera Omnia, Bâle, 1585.

48. Ibid., col. 1721a.

49. Ibid., col. 1719.

50. Cf. AGRIMI-CRISCIANI, Les « Consilia », op. cit., p. 104-105.

51. Toutefois, Arnaud (orientation qui paraît typique de la « ligne française ») prévoit seulement des consultations à deux et toujours in presentia : cf. Explicatio, op. cit., col. 1724 : si le médecin soignant a besoin d'un « auxilio alterius, sic ad alium peritiorem medicum recurrat et cum eo... conferet. Iste peritus si est propinquus, tunc aut satisfaciet... Non satisfaciet, et tunc recurrat ad alium distantem... " : entre-temps, le premier médecin soignant continuera ses visites et la thérapie, notant les nouveautés qui peuvent intervenir dans le cours [de la maladie].

52. Cf. Decem quaestiones, op. cit., p. 53-55. 
53. $C f$. Ps. ARISTOTE, Secretum secretorum (Secretum secretorum cum glossis et notulis... fratris Rogeri, éd. R. STEELE, Oxford, 1920 [Opera hactenus inedita Rogeri Baconi, V]), p. 59 : « O Alexander, in uno medico non confidas : quoniam unus medicus potens est ad nocendum... Si itaque potest fieri quod sint decem non sint minus, set fac eos convenire in unum. Et si sumenda fuerit cum medicina, noli sumere nisi de consilio plurimorum ».

54. Cf. HENRI DE MONDEVILle, op. cit., p. 66, 73.

55. Ibid., p. 115-16.

56. Ibid., p. 122.

57. Ibid., p. 127-28.

58. Cependant c'est le conseil en général commun et exprimé par tous les auteurs et par les médecins, déjà avant Henri, selon lequel on ne doit pas se disputer devant les laïcs : cf. MondEVILLE, op. cit., p. 61.

59. Henri en traite aussi dans la suite immédiate du texte, quand il enseigne sur le consilium palliativum : $c f$. p. 135-37.

60. $C f$. Agrimi-CRISCIANI, Les « Consilia », cit.

61. $C f$. E. WICKERSHEIMER, « Eine Diätregel für einen Bischof, ausgestellt von vier Professoren von Montpellier in der Mitte des 14. Jahrhunderts », Archiv für Geschichte der Medizin, 14, 1923, p. 184-86.

62. $C f$. E. WICKERSHEIMER, « Les secrets et les conseils de maître Guillaume Boucher et de ses confrères. Contribution à l'histoire de la médecine à Paris vers 1400 » (avec éd.), Bulletin de la Société française d'histoire de la médecine, 8, 1909, p. 199-305 ; D. JACQUART, La médecine médiévale, op. cit., passim.

63. $C f$. les conseils $47,87,88$ dans l'édition donnée par Wickersheimer.

64. Cf. C. CRISCIANI, "L'individuale nella medicina tra Medioevo e Umanesimo ", dans Umanesimo e medicina. Il problema dell'« individuale », R. CARDINI-M. REGOLIOSI dir., Rome, 1996, p. 1-20 ; V. NUTTON, « Case Histories in the Early Renaissance », dans The History of Case Histories, Stuttgart Conference, 1991 (non publié).

65. Cf. K. PARK, Doctors and Medicine in early Renaissance Florence, Princeton, 1985, p. $116 s q$.

66. $C f$. M. Nicoud, «Expérience de la maladie et échange épistolaire : les derniers moments de Bianca Maria Visconti (mai-octobre 1468) », Mélanges de l'École française de Rome. Moyen Âge, 112, 2000, p. 311-458 ; C. CRISCIANI, « Fatti, teorie, narratio e i malati a corte. Note sull'empirismo in medicina nel tardo medioevo ", Quaderni storici, 108/3, 2001, p. 659-717.

67. Cf. op. cit., Sermo primo, numérotation moderne, p. 16 a, 19 b, 20 ab.

68. M. McVAUGH, « Bedside Manners », loc. cit., p. 222. Sur ce thème, $c f$. aussi L. GARCíA BALLESTER, « Medical Ethics in Transition in the Latin Medicine of the Thirteenth and Fourteenth Centuries : New Perspectives on the Physician-Patient Relationship and the Doctor's Fee ", dans Doctors and Ethics: The earlier Historical Setting of Professional Ethics, A. WEAR- J. GEYER-KORDESCH-R. FRENCH dir., Amsterdam, 1993 (et d'autres articles dans ce même volume) ; CRISCIANI, «Infirmitas », cit. ; J. AGRIMI, C. CRISCIANI, « Charité et assistance ", loc. cit.

69. $C f$. par exemple (pour ce qui concerne la phase de l'enseignement) C. CRISCIANI, "Teachers and Learners in Scholastic Medicine : Some Images and Metaphors », History of Universities, 15, 1997-99, p. 75-101. 
70. $C f$. par exemple M. McVAUGH, "The Experimenta of Arnald of Villanova ", The Journal of Medieval and Renaissance Studies, 1, 1971, p. 107-18 ; ID., « Two Montpellier Recipe Collections ", Manuscripta, 20, 1976, p. 175-81.

71. Cf. HENRI DE MONDEVILLE, op. cit., p. 64-65.

72. Cf. TOMmaso Del GARBo, Summa medicinalis, Venise, $1506, \mathrm{f}^{\circ} 4 \mathrm{v}^{\circ} \mathrm{b}$; BARTOLOMEO Montagnana, Consilia, Venise, $1525, \mathrm{f}^{\circ} 25 \mathrm{r}^{\circ} \mathrm{a}$.

73. $C f$. C. CASAGRANDE, «Virtù della prudenza », loc. cit.

74. $C f$. C. CRISCIANI, "Valeurs éthiques et savoir médical entre le XII et le XIV siècle ", History and Philosophy of the Life Science, 5, 1983, p. 33-52 ; EAD., " Infirmitas ", loc. cit. ; J. AGRIMI, C. CRISCIANI, « Charité et assistance », loc. cit. ; $c f$. aussi Decem quaestiones, op. cit., p. 67 (« une conscience droite est la seconde chose requise du médecin, après la science droite $»)$.

75. AlBerTO DE ZANCARI, op. cit., p. 22.

\section{RÉSUMÉS}

Dare consilia est une des activités du médecin savant, formé à l'Université. Mise à part la tradition, typique des praticiens italiens, des consilia (écrits et formulés pour des patients), il est un acte médical important, la consultation, dans laquelle plusieurs médecins sont amenés à conférer sur l'état d'un malade, et à conseiller, pour finir, une procédure. Cette consultation est un des aspects du comportement du médecin qui n'est régulé ni par des normes corporatives ni par des décrets ecclésiastiques; en d'autres termes, elle fait partie des règles d'éthique ou « étiquette » que tout bon professionnel doit posséder. Sont analysées ici les règles que Henri de Mondeville, dans sa Chirurgia, établit pour cette réunion de médecins définie comme une collatio. Ses instructions sont confrontées tant avec celles que fournit Henri sur le comportement du médecin-chirurgien dans d'autres situations, qu'avec d'autres textes traitant de déontologie ou divers comptes rendus de consultation par des professionnels. Il en ressort que ces directives ou «conseils" d'Henri de Mondeville n'ont pas seulement pour finalité une plus grande efficacité de la pratique professionnelle, mais peuvent aussi être vus comme l'expression de l'éthique spécifique d'une communauté scientifique, celle des médecins du bas Moyen Âge.

Ethics of the consilia and of the consultation : about the moral cohesion of the medical profession (13th-14th centuries). Dare consilia is one of the activities of a learned, University trained physician. Except the tradition of the Consilia (written and formulated for patients who are not present), used by Italian practitioners, there is another important medical act, namely the consultation, whereby several physicians are brought in to discuss the state of a sick person and to advise on how to deal with the case. This consultation is one of the aspects of a physician's work that is regulated by neither corporative norms nor ecclesiastical decree; in other words it forms a part of ethical rules or "etiquette" which every professional should possess. This paper analyses the directives given by Henri de Mondeville in his Chirurgia, established for such a meeting of physicians and defined as a collatio. His instructions are compared with the rules he gives for the behaviour of the doctor-surgeon in other situations, as well as other texts dealing with the deontology or various consultative reports by professionals. It is evident that these directives or "counsels" of Henri de Mondeville not only aim for a more efficent professional 
practice, but can also be seen as the expression of professional ethics, specific to the scientific community of doctors of the late Middle Ages.

INDEX

Mots-clés : consultation, chirurgie, éthique professionnelle

Keywords : medical consultation, surgery, professional ethics, 14th century, Henri de Mondeville

\section{AUTEUR}

\section{CHIARA CRISCIANI}

Dipartimento di filosofia, Università di Pavia, piazza Botta 6, I-27100 Pavia 